\title{
MANUSEIO E SELEÇÃO DE ALIMENTOS EM TEMPOS DE COVID-19: RELATO DE EXPERIÊNCIA COM MULHERES REFUGIADAS
}

\author{
Juliana Vidal Vieira Guerra ${ }^{1}$ \\ Valdecyr Herdy Alves ${ }^{1}$ \\ Audrey Vidal Pereira ${ }^{1}$ \\ Rayanne Coco Cunha ${ }^{1}$ \\ Diego Pereira Rodrigues \\ Maria Clara Marques Fagundes ${ }^{3}$
}

\begin{abstract}
http://orcid.org/0000-0002-4509-1343
http://orcid.org/0000-0001-8671-5063

https://orcid.org/0000-0002-6570-9016

https://orcid.org/0000-0003-2093-2051

http://orcid.org/0000-0001-8383-7663

http: //orcid.org/0000-0003-4050-5655
\end{abstract}

Objetivo: Refletir sobre a experiência de pesquisadores em realizar entrevistas com mulheres refugiadas sobre cuidados com etapas de seleção, higiene e preparo de alimentos em tempos de Covid-19. Método: Trata-se de um relato de experiência a respeito do processo vivenciado por pesquisadores em manter coleta de informações a partir de entrevistas com mulheres refugiadas residentes no Estado do Rio de Janeiro sobre alimentação em tempos de pandemia. Resultados: Foram identificadas fragilidades para manter hábitos alimentares saudáveis, medidas de proteção individual e aquisição de informações seguras, o que contribuiu para reforçar a necessidade de orientações sobre segurança alimentar, atreladas às recomendações de isolamento social, lavagem das mãos e uso de máscaras, medidas importantes para o enfrentamento da pandemia e manutenção da vida. Conclusão: Essa experiência possibilitou criar grupos de orientações para as refugiadas na área da saúde alimentar, em especial no fornecimento de informações acerca de alimentos, seleção, higiene e preparo durante a pandemia. Descritores: Infecções por Coronavírus; Refugiados; Dieta; Segurança Alimentar e Nutricional; Preferências Alimentares.

\section{HANDLING AND SELECTION OF FOOD IN COVID-19 TIMES: REPORT OF EXPERIENCE WITH REFUGEE WOMEN}

Objective: Reflecting on the experience of researchers in conducting interviews with refugee women about food care due to steps of selection, hygiene and food preparation in times of COVID-19. Method: It is an experience report on the reflections regarding the process experienced by researchers in maintaining information collection from interviews with refugee women living in the state of Rio de Janeiro in times of pandemic. Results: reflecting on weaknesses to maintain healthy eating habits, individual protection measures and the acquisition of safe information contributed to reinforce the need for guidelines on food security, linked to recommendations for social isolation, hand washing and the use of important masks to face the pandemic and maintenance of life. Conclusion: This experience made it possible to create groups of guidelines for refugees in the area of food health, especially in providing information about food, selection, hygiene and preparation during the pandemic. Descriptors: Coronavirus Infections; Refugees; Diet; Food and Nutrition Security; Food Preferences.

\section{MANEJOY SELECCIÓN DE ALIMENTOS EN COVID-19 TIEMPOS: INFORME DE EXPERIENCIA CON MUJERES REFUGIADAS}

Objetivo: Reflexionar sobre la experiencia de los investigadores en la realización de entrevistas con mujeres refugiadas sobre la atención con pasos de selección, higiene y preparación de alimentos en tiempos de COVID-19. Metodo: Es un informe de experiencia sobre las reflexiones sobre el proceso experimentado por los investigadores para mantener la recopilación de información de entrevistas con mujeres refugiadas que viven en el estado de Río de Janeiro en tiempos de pandemia. Resultados: Reflexionar sobre las debilidades para mantener hábitos alimenticios saludables, las medidas de protección individual y la adquisición de información segura contribuyeron a reforzar la necesidad de pautas sobre seguridad alimentaria, vinculadas a recomendaciones para el aislamiento social, el lavado de manos y el uso de máscaras importantes para enfrentar la pandemia y mantenimiento de la vida. Conclusión: Esta experiencia permitió crear grupos de pautas para los refugiados en el área de la salud alimentaria, especialmente en el suministro de información sobre alimentos, selección, higiene y preparación durante la pandemia. Descriptores: Infecciones por Coronavirus; Refugiados; Dieta; Seguridad Alimentaria y Nutricional; Preferencias Alimentarias.

'Hospital Universitário Antônio Pedro, Universidade Federal Fluminense, Rio de Janeiro, RJ.

${ }^{2}$ Faculdade de Enfermagem, Universidade Federal do Pará, Belém, PA.

${ }^{3}$ Conselho Federal de Enfermagem, Brasilia, DF.

Autor Correspondente: Diego Pereira Rodrigues E-mail: diego.pereira.rodrigues@gmail.com Recebido: 30/4/2020 Aceito 01/6/2020 


\section{INTRODUÇÃO}

Até $\mathrm{Ol}$ de junho, a pandemia da COVID-19 atingiu quantitativo de 6.152.160 casos confirmados e mais de 371.700 mortes no mundo, sendo registrados no Brasil mais de 514.992 casos e 29.341 mortes. Antes mesmo da confirmação do primeiro caso no país, o Ministério da Saúde já havia decretado Emergência Sanitária, a partir da Lei no 13.979 de 6 de fevereiro de 2020 , sendo estabelecidas medidas de distanciamento do convivio social para a população em geral e quarentena para a população infectadal-2.

A crise mundial disparada pela COVID-19 coloca os refugiados em situações de grande risco, pois se trata de um dos grupos populacionais mais vulneráveis no mundo. O Alto Comissariado das Nações Unidas para Refugiados (ACNUR) sinaliza preocupação com estes ao redor do mundo, ciente de que muitos se encontram em lugares superlotados onde os serviços de saúde já se encontram sobrecarregados ${ }^{3-4}$.

O Brasil tem sido apontado como um país destino de populações migrantes refugiadas desde a deflagração das crises humanitárias ao redor do globo em países como Síria, Angola e Congo. No entanto, a partir de 2017, os reflexos na crise econômica e social na Venezuela intensificaram o aumento no número de solicitações de refúgio para o Brasil. Impulsionada pela tentativa de manter a integridade, escapando à situação de crise vivenciada nos países de origem, a população migrante, todavia, convive com barreiras no país destino, entre as quais o idioma e as dificuldades na comunicação, obstáculos no acesso a trabalho e renda, e a alimentação ${ }^{5}$.

A alimentação é uma necessidade básica humana, reconhecida na Declaração Universal dos Direitos Humanos, que também apresenta suas complexidades quando as pessoas se encontram em situação de refúgio forçado. Ademais, a Organização das Nações Unidas (ONU) propõe, nos Objetivos do Desenvolvimento Sustentável, especificamente o de número dois, uma meta para redução da fome mundial até $2030^{6-7}$.

Destaca-se, nesse âmbito, o papel da mulher, incluindo aquelas refugiadas, que historicamente têm sido responsáveis pelo fomento e preparo da alimentação no domicílio. Considerando o complexo e multidimensional fenômeno da alimentação que se desenvolve em torno da mulher refugiada, torna-se essencial refletir sobre os aspectos da alimentação em situação de refúgio, sobretudo quando em vigência de uma pandemia como a de coronavírus. O momento de emergência sanitária exige a necessidade de cuidados especiais com a alimentação, desde as etapas como a seleção dos alimentos até as etapas posteriores como cuidados higiênicos com o manuseio e preparo ${ }^{8}$.

Desde 2018 tem sido realizada investigação por pesquisadores do Grupo de Pesquisa Maternidade: Saúde da Mulher e da Criança, da Universidade Federal Fluminense (UFF) sobre alimentação de mulheres refugiadas, a partir de pesquisa intitulada "Transnacionalismo e Alimentação: história de vida de mulheres refugiadas". Este processo de pesquisa tem aproximado investigadores, além de fomentar a relação entre estudantes de graduação e pós-graduação no âmbito da saúde.

Assim, torna-se possível trazer à comunidade acadêmica um relato atualizado a respeito da experiência de entrevistar mulheres refugiadas com domicílio no Estado do Rio de Janeiro durante a pandemia de Covid-19. As entrevistas foram focadas nos cuidados com etapas de seleção, higiene e preparo de alimentos em tempos de COVID-19.

Pretende-se, neste estudo, compartilhar reflexões que emergiram no processo de realização de entrevistas com mutheres refugiadas, que têm que lidar com a seleção, manuseio e preparo de alimentos para suas famílias em condições de dificuldades de comunicação e insegurança alimentar e nutricional.

Acredita-se que o relato da experiência a das entrevistas de mulheres refugiadas, que necessitam levar a vida em paises atravessados por fatores sócio-culturais e econômicos diferentes daqueles que conheciam em seus países de origem, traz subsídios para compreender as especificidades da questão alimentar em tempos de pandemia junto a outras populações em situação de vulnerabilidade.

Desse modo, o estudo objetivou refletir sobre a experiência de pesquisadores em realizar entrevistas com mulheres refugiadas sobre cuidados com etapas de seleção, higiene e preparo de alimentos em tempos de COVID-19.

\section{MÉTODO}

\section{Tipo de Estudo}

Trata-se de um relato de experiência construído a partir do estudo intitulado "Transnacionalismo e Alimentação: histórias de vida de mulheres refugiadas", desenvolvido nas cidades de Niterói e Rio de Janeiro, inserido no Programa Acadêmico em Ciências do Cuidado em Saúde da Escola de Enfermagem Aurora de Afonso Costa (EEAAC) da UFF e vinculado ao Grupo de Pesquisa Maternidade: Saúde da Mulher e da Criança da mesma instituição.

A emergência da pandemia de COVID-19 tornou o contato com fins de pesquisa um processo complexo, sobretudo considerando que as pessoas se encontram em distanciamento sócio-linguístico-econômico-cultural e em situações de vulnerabilidade social quando em isolamento forçado em país diferente de sua origem.

\section{Cenário do Estudo}

Antes de ser declarado efetivamente o estado de emergência sanitária no país, a coleta de informações com entre- 
vistas narrativas era realizada a partir de encontros presenciais via Curso de Português para Refugiados, oferecido pela Universidade do Estado do Rio de Janeiro (UERJ) em parceria com a Cáritas Arquidiocesana do Rio de Janeiro.

Diante da emergência e aumento do número de casos de contágio pela COVID-19, decretado o isolamento social, este foi prorrogado, tornando o convívio social limitado à comunicação telefônica ou virtual. Visto que os autores permaneciam em isolamento social durante o mês de março de 2020, impossibilitando a realização de novas entrevistas narrativas presenciais, os contatos e encontros passaram a ser realizados de modo virtual por meio do aplicativo WhatsApp ${ }^{\circledR}$. A coleta de informações manteve foco no objetivo do projeto: identificar os valores culturais relativos à alimentação e nutrição da mulher refugiada a partir da história de vida delas. A pergunta norteadora que emergiu dos pesquisadores nas entrevistas virtuais foi: Como as mulheres refugiadas estão se organizando em relação à seleção e manuseio da alimentação?

Além de destacar pontos relacionados ao processo de coleta de informações on-line, cabe estabelecer um processo crítico e reflexivo a partir da literatura atualizada a respeito das informações que foram surgindo durante essa fase de isolamento social forçado, buscando tornar evidentes as dificuldades em manter diálogo sobre seleção e manuseio de alimentação por parte de mulheres refugiadas durante a pandemia.

Dessa maneira, é importante sinalizar que o processo analítico encontra-se em diálogo com as diretrizes do Ministério da Saúde em relação às medidas de enfrentamento estabelecidas em meio a pandemia, sobretudo com as recomendações sobre segurança alimentar e nutrição direcionadas à população em geral e, especificamente, à população de refugiados para o enfrentamento a COVID-19?.

\section{Período de Realização da Experiência}

A identificação das mulheres refugiadas participantes da pesquisa foi iniciada em janeiro de 2020, a partir da técnica snowball, sendo uma forma de compor uma amostra não probabilística de uma pesquisa ao identificar participantes iniciais, que indicam outros, que, por sua vez, indicam mais outros novos, e assim sucessivamente, até que seja encontrado um ponto de saturação das informações coletadas.

\section{Sujeitos envolvidos na experiência}

Participaram da experiência os pesquisadores, que são profissionais da saúde e uma estudante de graduação em Enfermagem, e as mulheres refugiadas. As reflexões da experiência dizem respeito ao processo experimentado pelos pesquisadores durante a produção e coleta de informações a partir de entrevistas com mulheres refugiadas residentes no
Estado do Rio de Janeiro em tempos de pandemia.

\section{Aspectos éticos}

Aprovado pelo Comitê de Ética em Pesquisa do Hospital Universitário Antônio Pedro da Universidade Federal Fluminense em janeiro de 2019, sob Parecer no 3.128.139, este projeto que utiliza narrativas de vida $^{10}$ de mulheres refugiadas sobre alimentação oportunizou compartilhar reflexões dos pesquisadores em tempos de COVID-19, a respeito do cuidado que estas mulheres têm com os alimentos. Vale destacar que a coleta de informações tem sido atravessada pelo período de pandemia. Nesse sentido, experimentar as entrevistas com aporte da narrativa de vida possibilitou um aprendizado significativo sobre mulheres refugiadas no cuidado com os alimentos em tempos de pandemia.

\section{OBJETIVO DA EXPERIÊNCIA}

Compartilhar reflexões da experiência de entrevista, tanto a respeito de etapas de seleção de alimentos referida por essas mulheres refugiadas, quanto sobre as condições de manuseio de alimentos para uma boa alimentação, a partir das narrativas das mulheres refugiadas, na pandemia de $\mathrm{CO}-$ VID-19.

\section{DESCRIÇÃO DA EXPERIÊNCIA}

A pandemia de COVID-19 apresentou uma nova dinâmica para a população. Entrevistas que eram realizadas presencialmente passaram a obedecer a uma nova realidade, a virtual com uso de aplicativos de comunicação. Para mulheres refugiadas em situação de isolamento forçado, a saída às ruas apenas se dá em situações de urgência e emergência ou para aquisição de produtos, como alimentos e medicamentos.

Esta realidade também influenciou a forma como a população seleciona e manuseia o preparo de alimentos no cotidiano. Estar num país diferente de sua origem pode ser mais um dos fatores que ampliam as dificuldades para manter alimentação saudável, pois situações econômicas e culturais podem ser decisivas para influenciar os modos de alimentação e as respectivas formas de manuseio dos alimentos. As mulheres refugiadas, em alguma medida referem sofrer influências nesse processo, tendo em vista os fatores relacionados ao convívio social incomum.

Os pesquisadores participantes da pesquisa conduziram as entrevistas com as mulheres refugiadas, por meio de contato telefônico ou por recursos digitais, possibilitando aos pesquisadores uma experiência interessante de coleta de informações importantes para a pesquisa

A preocupação com a possibilidade de contaminação se torna generalizada em uma situação de pandemia, podendo influenciar uma determinada medida pela preferência por ali 
mentos in natura, como verduras e legumes. Foi observado o consumo de alimentos de origem animal como carnes, enlatados e embutidos, baseado em crenças de que tais gêneros alimentícios seriam mais seguros para a saúde, práticos e evitariam o contágio. Os produtos sem necessidade de preparo e cocção, como biscoitos e pães, aparecem nas falas destas mulheres como alimentos que, além de permitirem minimizar custos, contribuem com a praticidade na rotina de alimentação da família.

Em determinada medida, a experiência demonstrou que mulheres refugiadas não deixaram de compartilhar a preocupação relacionada à tentativa de identificar alimentos que previnam o contágio da doença ou que protejam o sistema imune. Dentre os alimentos apontados pelas mulheres, foram ouvidos relatos de utilização de sucos de frutas cítricas com gengibre e alho, intencionando a melhora da imunidade, além da aquisição de chás, em especial de camomila e erva cidreira, que são alimentos ricos em compostos bioativos, como suporte de alimentação para supostamente "evitar o contágio". Assim, demonstram intenção de cuidar de si e dos familiares, com o uso de alimentos julgados adequados.

Esta experiência também demonstrou que o temor de contágio e a necessidade de prevenção despertaram maior busca por benefícios dos alimentos in natura, além de um maior consumo de fontes de vitaminas, minerais e fibras. No entanto, pode ser observado que as mulheres refugiadas necessitam do acesso a informações providas por profissionais da saúde quanto à seleção e às etapas de manuseio dos alimentos.

No caso do relato da utilização de chás, por exemplo, as mulheres entrevistadas relataram que o uso foi recomendado por pessoas do convívio social da população refugiada, sem orientação de profissional da saúde. De maneira geral, a experiência evidenciou o desconhecimento das mulheres refugiadas em relação ao manuseio e ao uso de alimentos para prevenção do risco de contaminação viral, o que em alguma medida pode contribuir para inseguranças alimentar e nutricional durante a pandemia.

Vale sinalizar que até o momento não existe evidência que sustente o uso de alimentos específicos na prevenção da COVID-19. No entanto, as recomendações da International Society for Immunonutrituion / Position Statement on Nutrition, Immunity and COVID-19 apontam o aporte de vitaminas, minerais e probióticos como estratégia de suporte nutricional e de suporte à imunidade dos pacientes, além de salientar que, no caso de contaminação pelo vírus, o estado nutricional deve ser avaliado em conjunto às múltiplas intervenções ${ }^{11}$.

No âmbito nacional, a assistência nutricional a população tem sido realizada de maneira geral, na modalidade on-line, a partir de projetos de extensão e pesquisa em Universidades Públicas e também a partir de recomendações específicas relacionadas ao novo coronavírus, como por exemplo, do Conselho Federal de Nutrição ${ }^{12}$.

Deste modo, torna-se interessante compartilhar algumas reflexões: Até que ponto as mulheres refugiadas têm conseguido acessar e utilizar informações seguras e atualizadas em relação à alimentação em tempos de COVID-19? As mulheres refugiadas têm sofrido influências da cultura brasileira no que diz respeito à seleção e manuseio de alimentos em tempos de pandemia? Em tempos de pandemia e em virtude de situações que se apresentem novas no dia a dia, torna-se imprescindivel que a população tenha acesso às informações atualizadas e de fontes seguras para autocuidado e proteção individual.

A Política Nacional de Alimentação e Nutrição (PNAN) possui como diretrizes a Promoção da Alimentação Adequada e Saudável e a Gestão das Ações de Alimentação e Nutrição. Para o alcance da alimentação saudável, no tocante à promoção da saúde, esta política aponta para a necessidade de processos de educação em saúde da população e de educação permanente para os trabalhadores da saúde ${ }^{13}$.

A transmissão de COVID-19 via alimentos ainda não é estabelecida na literatura científica. Entretanto, já se estabeleceu o tempo de sobrevida desse patógeno em superfícies como plásticos, vidro, papel e metal, sugerindo necessidade de higienização com água e sabão, álcool a 70\% ou, ainda, solução de hipoclorito de sódio 0,1\% destas superfícies que entram em contato com os alimentos a fim de conter contaminação ${ }^{14}$.

Em relação à alimentação, com destaque para a seleção e manuseio dos alimentos, pode-se observar que mulheres refugiadas não conseguem vivenciar práticas de consumo consciente, tendo dificuldade também para manter vigilância com a higiene, conforme preconizam as orientações institucionais $^{12}$. Logo, observa-se maior predisposição ao risco de contágio, levando-se em conta possiveis dificuldades econômicas, de comunicação e de acesso à informação atualizada.

As mulheres refugiadas que trabalham em serviços essenciais como supermercados, por exemplo, estão predispostas às fragilidades relacionadas às medidas de proteção individuais. Apesar de demonstrarem conhecimento quanto à importância do uso do álcool gel quando em transportes públicos, não se atentam, por exemplo, para o risco do contato com superfícies que possam estar contaminadas no serviço, tampouco para a necessidade de higienizar pacotes de alimentos que levam para o domicílio. Não somente demonstram dificuldades para manter vigilância quanto à proteção individual e diminuição do risco de contaminação via manu- 
seio de alimentos, como também compartilham dificuldades para priorizar processos que garantam a manutenção de alimentação saudável durante a pandemia.

A premissa de utilizar alimentos que beneficiem a saúde não se encontra atrelada às reais possibilidades de acesso e manutenção de hábitos saudáveis de alimentação. Todavia, como tentativa de proteção, sinalizaram a opção por alimentos enlatados e embutidos, com base na crença de que seu consumo seria mais seguro em tempo de COVID-19, o que, de certo modo, interfere também na manutenção de alimentação saudável. Neste ponto, vale ressaltar que, mediante necessidade de proteção da vida, os pesquisadores também realizavam, após a entrevista, orientações relacionadas à pandemia no momento de contato com as mulheres, não se restringindo à escuta de investigação.

O cuidado com os alimentos, como elemento central na segurança alimentar e nutricional, requer conhecimento prévio de higiene individual e para com os alimentos como uma forma de prevenção de surtos veiculados, desde a seleção de alimentos nos estabelecimentos comerciais até o armazenamento e preparo no domicílio. Observou-se, na experiência, dificuldade para garantir práticas vigilantes de higiene necessárias para o manuseio de alimentos no domicílio em tempos de pandemia ${ }^{14}$

Com relação ao preparo dos alimentos, a recomendação é de tratamento térmico (cocção ou cozimento) atingindo temperatura de $70 \cong \mathrm{C}$ em todas as partes destes, reforçando o cuidado e a prevenção do contágio ${ }^{15}$. No entanto, verificou-se que a única preocupação era com cozimento sem instruções específicas, não se observando a preocupação em se atingir temperaturas elevadas em todo o alimento. Também foi constatada a falta de acesso a informações com relação aos alimentos in natura e à necessidade de ações de higiene utilizando solução de hipoclorito de sódio, conforme preconizado pelo Ministério da Saúde. Foram, ainda, observados relatos somente do uso de água ou sabão como medida para higienizar frutas, legumes e vegetais. Sobre alimentos embalados, os pesquisadores observaram a inexistência da preocupação com as embalagens plásticas e utilização de álcool gel ou pano umedecido em superfície como forma de higiene. Todavia, cabe refletir o quanto é difícil manter tais medidas levando-se em conta restrições econômicas e de tempo individual no dia a dia.

Além disso, foi possivel observar que as mulheres refugiadas referem que acessam informações relacionadas às medidas preventivas ao contágio veiculadas nos meios de comunicação. Interessante refletir que, por vezes, estas informações têm sido divergentes, além de serem permeadas por notícias falsas e vagas.
Deste modo, em alguma medida, as mulheres refugiadas estão sob o estado de insegurança alimentar e nutricional, uma vez que o acesso a alimentos de modo seguro não tem sido uma realidade durante o período de pandemia. Assim, torna-se imprescindivel que essas mulheres tenham conhecimento tanto da necessidade de se protegerem de possível contaminação em momentos de seleção de alimentos, quanto da importância de manter uma alimentação saudável, não somente com vistas à proteção de doenças crônico-degenerativas, como também para a manutenção de imunidade enquanto fator de sustentação da vida.

\section{PRINCIPAIS RESULTADOS ALCANÇADOS}

A experiência possibilitou aos pesquisadores:

- A criação de grupos de orientações para as refugiadas na área da saúde alimentar, especial no fornecimento de informações acerca de alimentos, seleção, higiene e preparo durante a pandemia de COVID-19;

- Identificar não somente os alimentos de preferência dessas mulheres, mas também a relação delas com os alimentos e o processo de seleção e manuseio destes durante a pandemia.

\section{Limitações para a experiência}

A principal limitação do estudo está relacionada com o contato, que deixou de ser presencial e passou a ser vivenciado por meio do aplicativo WhatsApp®, ao qual não é de acesso a todas as participantes.

\section{Considerações para a prática}

Indo ao encontro das diversas estratégias de enfrentamento e mitigação da pandemia, torna-se fundamental destacar a necessidade da criação de um programa multiprofissional de suporte e orientação nutricional. Garantir educação em saúde, atendendo em especial populações em situação de vulnerabilidade, sobretudo em isolamento do convívio social, contribui para veicular informações fidedignas e seguras em tempos de instantaneidade da comunicação.

\section{CONSIDERAÇÕES FINAIS}

A experiência vivenciada pelos pesquisadores durante as entrevistas com aporte nas narrativas de vida foi de valiosa contribuição para o andamento da pesquisa. Ao observar os variados aspectos que abrangem a alimentação e a nutrição da população refugiada numa situação de risco à saúde em meio à pandemia de COVID-19, pode-se refletir que existe um estado de insegurança alimentar, além de demonstrar fragilidades relacionadas ao processo de compartilhar informações com foco nos processos de seleção, manuseio e preparo dos alimentos. 
Deste modo, informação atualizada de origem institucional, traduzida para o idioma de origem, poderá, em alguma medida, garantir maior segurança quanto à seleção e manuseio dos alimentos em tempo de emergência sanitária/pandemia. Ampliar o escopo de orientações especificas na área da segurança alimentar e nutricional tem sido tão necessário quanto reforçar as orientações de isolamento social, lavagem das mãos e uso de máscaras para o enfrentamento da pandemia e manutenção da saúde e da vida.
Contribuições dos Autores: Concepção e/ou desenho do estudo: Juliana VVG, Alves VH, Pereira AV, Cunha RC, Rodrigues DP, Fagundes MCM; Coleta, análise e interpretação dos dados: Juliana VVG, Alves VH, Pereira AV, Cunha RC, Rodrigues DP, Fagundes MCM; Redação e/ou revisão crítica do manuscrito: Juliana VVG, Alves VH, Pereira AV, Cunha RC, Rodrigues DP, Fagundes MCM; Aprovação da versão final a ser publicada: Juliana VVG, Alves VH, Pereira AV, Cunha RC, Rodrigues DP, Fagundes MCM.

\section{REFERÊNCIAS}

1. Word Health Organization (WHO). Coronavirus disease 2019 (COVID-19) Situation Report - 59. Publicado em 19 de Março de 2020. [Internet]. 2020 [cited 2020 april 25]. Available from: https://apps.who.int/iris/bitstream/handle/10665/331597/nCoVsitrep19Mar2020-eng.pdf https://covid19. who.int/.

2. Governo Federal (BR). Lei no 13.979, de 6 de fevereiro de 2020. Medidas para enfrentamento da emergência em saúde pública de importância internacional decorrente do coronavirus responsável pelo surto de 2019. Diário Oficial da União. [Internet]. 2020 [cited 2020 april 25 ]. Available from: http://www.in.gov.br/en/web/dou/-/lei-n-13.979-de-6-de-fevereiro-de-2020-242078735.

3. The Lancet. COVID-19 will not leave behind refugees and migrants. The Lancet. [Internet]. 2020 [cited 2020 april 25]; 395(10230):1090. Available from: https://www.thelancet.com/journals/lancet/article/PIISO140-6736(20)30758-3/fulltext.

4. United Nations High Commissioner for Refugees (UNHCR). UN Refugee Agency steps up COVID-19 preparedness, prevention and response measures. [Internet]. 2020 [cited 2020 april 25]. Available from: https://www.unhcr.org/uk/news/press/2020/3/5e677f634/un-refugee-agency-steps-covid-19-preparedness-prevention-response-measures.html

5. Cazella, LG et al. Qualidade de vida de mulheres e as características sociodemográficas associadas. Enfermagem em Foco [Internet]. 2019 [cited 2020 may 29]; v. 10, n. 3. Available from: http://revista.cofen.gov.br/index.php/enfermagem/article/view/2448.

6. Ministério da Saúde (BR). Secretaria de Atenção à Saúde. Departamento de Atenção Básica. Política Nacional de Alimentação e Nutrição. 1. ed. 1. reimpr. Brasilia: Ministério da Saúde; 2013. [Internet] 2013 [cited 2020 Apr 25]. Available from: https://bvsms.saude.gov.br/bvs/publicacoes/politica_nacional_alimentacao_nutricao.pdf

7. Organização das Nações Unidas (ONU). Roteiro para a Localização dos Objetivos de Desenvolvimento Sustentável: Implementação e Acompanhamento no nivel subnacional. Global Taskforce of local and regional governments. [Internet] 2016. [cited 2020 Apr 25]. Available from: https:// nacoesunidas.org/wp-content/uploads/2017/06/Roteiro-para-a-Localizacao-dos-ODS.pdf.

8. SawayaAL, PelianoAM,Albuquerque MP, Domene SMA.Afamilia e o direito humanoàalimentação adequada e saudável. Estudos Avançados [Internet] 2019 [cited 2020 Apr 25]; 33(97):361-382. Available from: https://www.scielo.br/scielo.php?script=sci_arttext\&pid=S0103-40142019000400361.

9. Oliveira TC, Abranches MV, Lana RM. (In)Segurança alimentar no contexto da pandemia por SARS-CoV-2. Cad Saúde Pública [Internet]. 2020 [cited 2020 Apr 25]; 36(4):e00055220. Available from http://www.scielo.br/scielo.php?script=sci_arttextEpid=S0102-311X2020000400501\&lng=en\&nrm=iso

1. Daniel Bertaux. Le récit de vie. Paris, France: Armand Collin; 2017. 132p.

10. International Society for Immunonutrition (ISIN). ISIN Position Statement on Nutrition, Immunity and COVID-19. [Ifnternet]. 2020 [cited 2020 Apr 25]. Available from: http://www.immunonutrition-isin.org/docs/isinComunicadoCovid19.pdf.

2. Conselho Federal de Nutricionistas (CFN). Recomendações do CFN: boas práticas para atuação do nutricionista e do técnico em nutrição e dietética durante a pandemia de coronavirus. [Internet]. 2020 [cited 2020 Apr 25]. Available from: https://www.cfn.org.br/wp-content/uploads/2020/03/ nota_coronavirus_3-1.pdf

11. Ministério da Saúde (BR). Portaria no 2.076, de 5 de agosto de 2019. Incentivo para estruturação e implementação de ações de alimentação e nutrição, com base na Política Nacional de Alimentação e Nutrição (PNAN). Brasilia: Ministério da Saúde; 2019. [Internet]. 2019 [cited 2020 Apr 25]. Available from: https://brasilsus.com.br/wp-content/uploads/2019/08/portaria2076.pdf

12. Van Doremalen N, Bushmaker T, Morris D, Holbrook M, Gamble A, Williamson B, et al. Aerosol and surface stability of HCoV-19 (SARS-CoV-2) compared to SARS-CoV-1. MedRxiv [Internet]. 2020 [cited 2020 Apr 25]. Available from: https://www.ncbi.nlm.nih.gov/pmc/articles/PMC7121658/pdf/ NEJMc2004973.pdf.

13. Kampf G, Todt D, Pfaender S, Steinmann E. Persistence of coronaviruses on inanimate surfaces and their inactivation with biocidal agents. $J$ Hosp Infect [Internet]. 2020 [cited 2020 Apr 25]; 104(3): 246-51. Available from: https://www.journalofhospitalinfection.com/article/SO195$6701(20) 30046-3 /$ fulltext. 